\title{
A matrix of consonant-cluster-free monosyllabic words in English
}

\author{
NELSON COWAN \\ University of Missouri, Columbia, Missouri
}

\begin{abstract}
In language-related psychological research, it is often necessary to search for sets of words with certain well-controlled phonological properties. To aid in searches of this kind, this paper presents a matrix of consonant-cluster-free, monosyllabic English words that are classified according to their phonemes. The matrix is of considerable use in the construction of experimental stimuli. Its applications are discussed.
\end{abstract}

A frequent, masochistic activity in linguistically related psych Jlogy is to search for sets of stimulus words having certain systematic properties (phonological, orthographic, semantic, etc.). Construction of these word sets typically involves an open-ended mental search, often precipitating an extended episode of the tip-of-the-tongue state discussed by Brown and McNeill (1966). Clearly, a more systematic solution is desirable whenever the stimulus design problem is a recurrent one and the solution space is manageably small. The solution space to one such problem is provided in the Appendix, the main contribution of the present paper. The Appendix includes, in matrix form, all monosyllabic English words with simple CVC (consonant-vowel-consonant), CV, VC, or V phoneme structures (i.e., all monosyllabic words except words with consonant clusters). The listing can aid in the construction of sets of either phonologically uniform or phonologically varied word or nonword syllables. After the listing is described further, several examples of its application will be provided, and its limits will be discussed.

\section{CONSTRUCTION OF THE MATRIX}

The matrix (see Appendix) is based on the phonemes (sounds) of English, not the letters used to represent these sounds. In each section of the Appendix, the initial consonant is listed in the leftmost column, and the vowel sound is listed in the top row. The sections of the Appendix differ according to the final consonant. Diphthongs (the compound vowels [ar] as in find, [ $\mathrm{r}]$ as in boil, and [au] as in down) and affricates (the compound consonants [dz] as in judge and [t]] as in church) are included, because there is evidence that they are generally perceived as single units (Cowan, Braine, \& Leavitt, 1985). The nonlinguist can decipher the phonetic symbols by reading the words occurring in each row or column. The sym-

I thank Jean Ispa, Scott Saults, Cristy Cartwright, and William Ganong for comments on an earlier draft of this manuscript.

Address reprint requests to Nelson Cowan, Department of Psychology, University of Missouri, 210 McAlester Hall, Columbia, MO 65211. bol " $\varnothing$ " is used to represent the cases in which there is no initial or final consonant (i.e., in $\mathrm{CV}, \mathrm{VC}$, or $\mathrm{V}$ structures).

If a particular combination of phonemes does not form an English word, that cell of the table is left blank. For each set of homophonic words (e.g., real vs. reel), only one word is recorded, and words with two possible pronunciations (e.g., vase, pronounced [vez] or [vaz]) appear in two locations in the table. Slang words, common first names, and contractions are included, but are presented in parentheses. Finally, because of regional variations and individual differences in speech and English usage, the table cannot be totally accurate for all speakers, and it represents my dialect (Washington, DC area speech with influences in the past 15 years of New England and several midwestern states). A much larger table of monosyllabic and some disyllabic words, phonetically arranged according to a standard British dialect, also exists (Rockey, 1973).

\section{SOME APPLICATIONS OF THE MATRIX}

Investigators of speech perception often use sets of simple monosyllables as the stimuli (see, e.g., the classic readings edited by Lehiste, 1967). In these studies, it is often the case that some of the syllables form words and some do not. Although it is generally the phonetic properties of the syllables that are of interest in these studies, lexical recognition of some items might influence the results. To avoid this problem, the Appendix presented here makes it easier to determine whether, for a particular study, it would be possible to use a set of syllables entirely of word status or of nonword status.

It is sometimes necessary to devise stimuli containing matching phonetic properties in word and nonword sets. For example, Ganong (1980) and Fox (1984) examined the effects of lexical status on phoneme identification. They presented voiced-voiceless word pairs in which only the voiced member was a word (e.g., dust vs. tust) or only the voiceless member was a word (e.g., dest vs. test), and in Fox's study, pairs in which both or neither mem- 
ber was a word. The Appendix yields an exhaustive set of candidate stimuli that could be used to design studies such as these.

Several of my studies of speech memory further illustrate how the listing can be used. First, Cowan and Kielbasa (1986) presented young children with lists of nonsense syllables in order to examine memory for speech sounds. After each list, there was a variable silent delay period filled with a distracting task, and then the child was to respond by selecting pictures whose names rhymed with syllables on the spoken list (e.g., by pointing to a bed in response to the syllable ged). For this study, the matrix in the Appendix aided in finding the best word and nonword stimuli with similar phonetic properties. Second, in a study of young children's speech memory currently under way, it was necessary to find a set of monosyllabic words with a CV structure that could be scored independently for correct retention of the consonant and vowel. The matrix yielded a four-word set, including bee, tea, bow, and toe. Because the options can be examined exhaustively, this stimulus set can be used with the assurance that a better set of stimuli suitable for the children is not likely to be found.

A more detailed example can demonstrate the potential power of the Appendix, but some background is needed to appreciate the design problem. In an important paper on speech memory development, Conrad (1971) found that school-aged children (e.g., 8-year-olds) remembered sets of pictures much better when the pictures had acoustically dissimilar names (fish, spoon, hat, etc.) than when they had similar names (bat, cat, hat, etc.). In contrast, little effect of acoustic similarity was obtained in preschool children (e.g., 4-year-olds). It was thought that the developmental difference was in verbal labeling, but Hulme (1984) obtained a comparable effect when the same items were presented as spoken words. In the author's laboratory, this effect has been replicated with adults and 4-year-old children. The data appear to indicate that instead of a development merely in verbal labeling, there is an interesting development in the processing of speech between the preschool and school years.

However, the stimuli used in these studies include several complicating factors that make it impossible to specify the nature of the developing speech processes. First, the dissimilar words were phonetically longer than the similar words, so that word similarity and length were confounded. Second, the acoustic similarities occurred in both the vowel and the final consonant, and the contribution of each is unclear. If the basis of the similarity effect is in sensory memory, it should occur only if there is vowel similarity (Cowan, 1984; Crowder, 1971; M. J. Watkins, O. C. Watkins, \& Crowder, 1974), whereas if an articulatory memory is involved, the effect should occur with either vowel or consonant similarity. With the help of the Appendix, better stimulus sets have been devised for a further study, described below.
Table 1

Series of Words for an Experiment on the Effects of Consonant Similarity on Word Span

1. seed, said, sad, sod, sawed, sowed, sued, side

2. neat, knit, net, gnat, not, nut, note, night

3. deem, dim, dame, dam, dumb, dome, doom, dime

4. keep, kip, cape, cap, cop, cup, cope, coop

5. lick, lake, lock, luck, look, like, leak, lack

6. miss, mace, mess, mass, muss, moss, moose, mouse

7 . bees, bays, buzz, bows, booze, buys, boys, boughs

8. peel, pill, pail, pal, pole, pull, pool, pile

The aim is to determine the nature of the acoustic similarity effect by repeating the Hulme (1984) procedure with adult subjects only, but with better controlled stimuli. Consonant and vowel similarity are to be manipulated in separate experiments, using stimuli constructed with the help of the Appendix. Illustrating these, Table 1 contains sets of words that can be used to examine the effects of intralist consonant similarity. Each of the eight sets of words contains eight items with the same consonants but different vowels. Stimulus lists are to be constructed using words within a set, for the acoustically similar condition (e.g., neat, knit, net, gnat, not, nut, note, night), or using only one word from each set, each with a different vowel, for the acoustically dissimilar condition (e.g., seed, knit, mess, cap, lock, buzz, pole, dime). Thus, across trials the same words are to be used in the acoustically similar and dissimilar conditions, but with the words grouped into lists differently in these conditions. Construction of this stimulus set and of other stimuli to examine the effects of vowel similarity would not have been feasible without the Appendix.

Finally, the Appendix might be of use to linguists (e.g., Bhat, 1978; Vihman, 1978) attempting to characterize the rules and tendencies of phoneme combination. An important aspect of the Appendix that enables all of these applications is the use of a matrix form. Rhyming dictionaries (e.g., Stein, 1960) provide a much wider variety of word types, but they are not appropriately organized for a systematic, phoneme-by-phoneme search.

\section{LIMITS OF THE APPENDIX}

Although the CVC solution space is relatively small (with 7,360 cells in the Appendix), the solution space for all monosyllabic English words is considerably larger. Based on descriptions of consonant clusters provided by Bloomfield (1933, pp. 127-138) and Greenberg (1978), it can be shown that a matrix accommodating all monosyllabic English words contains over 77,000 cells (cf. Rockey, 1973).

The Appendix is arranged best for comparisons of word sets with a common last consonant, whereas comparisons of words with different last consonants are somewhat less convenient. However, the entire matrix could be entered into a computer base to permit various types of searches. Alternatively, when one wishes to compare sections of 
the Appendix (i.e., words with different last consonants) with less effort, it has proven helpful to photocopy the Appendix sections onto clear plastic to permit viewing of several superimposed sections.

\section{REFERENCES}

Bhat, D. H. S. (1978). A general study of palatalization. In J. H. Greenberg (Ed.), Universals of human language. Volume 2: Phonology (pp. 47-92). Stanford, CA: Stanford University Press.

BLOOMFIELD, L. (1933). Language. New York: Holt, Rinehart, Winston. Brown, R., \& MCNEILL, D. (1966). The "tip of the tongue" phenomenon. Journal of Verbal Learning \& Verbal Behavior, 5, 325-337.

Conrad, R, (1971). The chronology of the development of covert speech in children. Developmental Psychology, 5, 398-405.

Cowan, N. (1984), On short and long auditory stores. Psychological Bulletin, 96, 347-370.

Cowan, N., Braine, M. D. S., \& LeavitT, L. A. (1985). The phonological and metaphonological representation of speech: Evidence from fluent backward talkers. Journal of Memory \& Language, 24, 679-698.

Cowan, N., \& KiElBasa, L. (1986). Temporal properties of memory for speech in preschool children. Memory \& Cognition, 14, 382-390.

Crowder, R. G. (1971). The sound of vowels and consonants in immediate memory. Joumal of Verbal Learning \& Verbal Behavior, 10, 587-596.
Fox, R. A. (1984). Effect of lexical status on phonetic categorization. Journal of Experimental Psychology: Human Perception \& Performance, 10, 526-540.

GANONG, W. F. (1980). Phonetic categorization in auditory word perception. Journal of Experimental Psychology: Human Perception \& Performance, 6, 110-125.

GREENBERG, J. H. (1978). Some generalizations concerning initial and final consonant clusters. In J. H. Greenberg (Ed.), Universals of human language. Volume 2: Phonology (pp. 243-279). Stanford, CA: Stanford University Press.

Hulme, C. (1984). Developmental differences in the effects of acoustic similarity on memory spans. Developmental Psychology, 20, 650-652.

LEHISTE, I. (1967). Readings in acoustic phonetics. Cambridge, MA: MIT Press.

RoCKEY, D. (1973). Phonetic lexicon of monosyllabic and some disyllabic words, with homophones, arranged according to their phonetic structure. London: Heyden \& Son.

STEIN, J. (1960). Rhyming dictionary. New York: Random House.

VIHMAN, M. M. (1978). Consonant harmony: Its scope and function in child language. In J. H. Greenberg (Ed.), Universals of Human Language. Volume 2: Phonology (pp. 281-334). Stanford, CA: Stanford University Press.

Watkins, M. J., Watkins, O. C., \& Crowder, R. G. (1974). The modality effect in free and serial recall as a function of phonologial similarity. Journal of Verbal Learning \& Verbal Behavior, 13, 430-447.

\section{APPENDIX \\ A Matrix of Consonant-Cluster-Free Monosyllabic Words}

Exemplary words from each of the $\mathbf{2 0}$ sections, in order of each section's appearance in this listing, are: bib, bead, big, beep, beet, beak, beam, bean, king, deal, beer, fizz, guess, dish, give, beef, teeth/teethe, page/beige, beach, bee.

\begin{tabular}{|c|c|c|c|c|c|c|c|c|c|c|c|c|c|c|c|c|}
\hline \multirow{2}{*}{$\begin{array}{c}\text { First } \\
\text { Consonant }\end{array}$} & \multicolumn{16}{|c|}{ Vowel } \\
\hline & $\mathbf{i}$ & I & e & $\varepsilon$ & $\not{x}$ & a & $\Lambda$ & 0 & 0 & U & $\mathbf{u}$ & aI & II & au & ju & 3 \\
\hline & \multicolumn{16}{|c|}{ Words Ending in b } \\
\hline b & - & bib & (Babe) & - & - & (Bob) & (bub) & - & - & - & (boob) & - & - & - & - & (burb) \\
\hline d & - & - & - & (deb) & dab & - & dub & daub & - & - & - & - & - & - & - & - \\
\hline 8 & - & - & - & - & gab & gob & - & - & - & - & - & - & - & - & - & - \\
\hline p & - & - & - & - & - & - & pub & - & - & - & - & - & - & - & - & - \\
\hline$t$ & - & - & - & - & tab & - & tub & - & - & - & tube & - & - & - & - & - \\
\hline $\mathbf{k}$ & - & - & - & - & cab & cob & cub & - & - & - & - & - & - & - & cube & curb \\
\hline $\mathbf{m}$ & - & - & - & - & - & mob & - & - & - & - & - & - & - & - & - & - \\
\hline $\mathrm{n}$ & - & nib & - & - & nab & knob & nub & - & - & - & - & - & - & - & - & - \\
\hline 1 & - & - & - & - & (lab) & lob & - & - & lobe & - & (lube) & - & - & - & - & - \\
\hline$r$ & - & rib & - & (Reb) & - & rob & nub & - & robe & - & (Rube) & - & - & - & - & - \\
\hline j & - & - & - & - & - & - & - & - & - & - & - & - & - & - & - & - \\
\hline$z$ & - & - & - & - & - & - & - & - & - & - & - & - & - & - & - & - \\
\hline $\mathbf{s}$ & - & (sib) & - & - & - & sob & (sub) & - & - & - & - & - & - & - & - & Serb \\
\hline s & - & - & - & - & - & - & - & - & - & - & - & - & - & - & - & - \\
\hline $\mathbf{v}$ & - & - & - & - & - & - & - & - & - & - & - & (vibe) & - & - & - & verb \\
\hline $\mathbf{f}$ & - & fib & - & - & - & fob & - & - & - & - & - & - & - & - & - & - \\
\hline h & - & - & - & - & - & hob & hub & - & - & - & - & - & - & - & - & (Herb) \\
\hline d & - & - & - & - & - & - & - & - & - & - & - & - & - & - & - & - \\
\hline$\theta$ & - & - & - & - & - & - & - & - & - & - & - & - & - & - & - & - \\
\hline$w$ & - & - & - & web & - & - & - & - & - & - & - & - & - & - & - & - \\
\hline$d 3$ & - & $\mathrm{jib}$ & - & - & jab & job & - & - & (Job) & - & - & jibe & - & - & - & - \\
\hline if & - & - & - & - & - & - & chub & - & - & - & - & - & - & - & - & - \\
\hline (ø) & - & - & - & ebb & - & - & - & - & - & - & - & - & - & - & - & herb \\
\hline
\end{tabular}




\begin{tabular}{|c|c|c|c|c|c|c|c|c|c|c|c|c|c|c|c|c|}
\hline \multirow{2}{*}{$\begin{array}{c}\begin{array}{c}\text { First } \\
\text { Consonant }\end{array} \\
\end{array}$} & \multicolumn{16}{|c|}{ Vowel } \\
\hline & $\mathrm{i}$ & I & e & $\varepsilon$ & $æ$ & $\mathbf{a}$ & $\Lambda$ & 2 & o & $u$ & $\mathbf{u}$ & aI & II & au & ju & 3 \\
\hline & \multicolumn{16}{|c|}{ Words Ending in d } \\
\hline$b$ & bead & bid & bade & bed & bad & (bod) & bud & (baud) & (bode) & - & - & bide & buoyed & bowed & - & bird \\
\hline$d$ & deed & did & - & dead & (Dad) & - & dud & - & - & - & (dude) & died & - & - & - & - \\
\hline $\mathrm{g}$ & - & - & - & - & gad & god & - & - & goad & good & - & guide & - & - & - & gird \\
\hline $\mathbf{p}$ & peed & - & paid & - & pad & pod & - & pawed & - & - & - & pied & - & - & - & purred \\
\hline$t$ & teed & - & - & (Ted) & (tad) & (Tod) & - & - & toad & - & - & tide & toyed & - & - & (turd) \\
\hline k & keyed & kid & cade & - & cad & $\operatorname{cod}$ & cud & cawed & code & could & cooed & - & - & cowed & cued & curd \\
\hline $\mathrm{m}$ & mead & mid & made & - & $\operatorname{mad}$ & $(\mathrm{mod})$ & mud & - & mode & - & $\operatorname{mood}$ & - & - & - & mewed & - \\
\hline $\mathbf{n}$ & need & - & neighed & (Ned) & - & nod & - & gnawed & node & - & nude & - & - & - & - & (nerd) \\
\hline 1 & lead & lid & layed & led & lad & - & - & - & load & - & lewd & lied & (Lloyd) & loud & - & - \\
\hline$r$ & read & rid & raid & red & $\mathrm{rad}$ & rod & - & - & road & - & rude & ride & - & - & - & - \\
\hline $\mathbf{j}$ & - & (Yid) & - & - & - & - & - & yawed & - & - & (you'd) & - & - & - & - & - \\
\hline $\mathbf{Z}$ & - & - & - & zed & - & - & - & - & - & - & - & - & - & - & - & - \\
\hline $\mathrm{s}$ & seed & (Sid) & - & said & sad & sod & sudd & sawed & sowed & - & sued & side & - & - & - & surd \\
\hline f & (she'd) & - & shade & shed & shad & shod & - & - & showed & should & shooed & shied & - & - & - & - \\
\hline v & - & - & - & - & - & - & - & - & - & - & - & vied & void & vowed & viewed & - \\
\hline f & feed & fid & fade & fed & fad & - & - & - & - & - & food & - & - & - & feud & furred \\
\hline $\mathrm{h}$ & heed & hid & - & head & had & hod & - & - & hoed & hood & (who'd) & hide & - & (how'd) & - & heard \\
\hline$\delta$ & - & - & (they'd) & - & - & - & - & - & - & - & - & - & - & - & - & - \\
\hline$\theta$ & - & - & - & - & - & - & thud & thawed & - & - & - & - & - & - & - & third \\
\hline$w$ & weed & - & wade & wed & - & wad & - & - & woad & would & wooed & wide & - & (wowed) & - & word \\
\hline d3 & - & - & jade & - & - & - & - & jawed & - & - & (Jude) & - & - & - & - & - \\
\hline ts & - & chid & - & - & (Chad) & - & - & - & - & - & chewed & chide & - & (chowed) & - & - \\
\hline (ø) & - & id & aid & $(E d)$ & add & odd & - & awed & owed & - & - & eyed & - & - & (you'd) & - \\
\hline
\end{tabular}

\begin{tabular}{|c|c|c|c|c|c|c|c|c|c|c|c|c|c|c|c|c|}
\hline \multirow{2}{*}{$\begin{array}{c}\text { First } \\
\text { Consonant }\end{array}$} & \multicolumn{16}{|c|}{ Vowel } \\
\hline & 1 & I & e & $\varepsilon$ & $\approx$ & a & $\mathbf{A}$ & 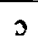 & 0 & $u$ & $\mathbf{u}$ & aI & II & au & ju & 3 \\
\hline
\end{tabular}

\section{Words Ending in $\mathbf{g}$}

\begin{tabular}{|c|c|c|c|c|c|c|c|c|c|c|c|c|c|c|c|c|}
\hline b & - & big & - & beg & bag & - & bug & bog & - & - & - & - & - & - & - & (burg) \\
\hline d & - & dig & - & - & - & - & dug & $\operatorname{dog}$ & - & - & - & - & - & - & - & - \\
\hline $\mathbf{g}$ & - & (gig) & - & - & gag & - & - & - & - & - & - & - & - & - & - & - \\
\hline p & - & pig & - & peg & - & - & pug & - & - & 一 & - & - & - & - & - & - \\
\hline$t$ & - & - & - & - & tag & - & tug & - & - & - & - & - & - & - & - & - \\
\hline k & - & - & - & keg & - & $\operatorname{cog}$ & - & - & - & - & - & - & - & - & - & - \\
\hline m & - & (Mig) & - & (Meg) & - & - & mug & - & - & - & (Moog) & - & - & - & - & - \\
\hline $\mathbf{n}$ & - & - & - & - & nag & nog & - & - & - & - & - & - & - & - & - & - \\
\hline 1 & league & - & - & leg & lag & - & lug & $\log$ & - & - & - & - & - & - & - & - \\
\hline$r$ & - & rig & - & - & rag & - & rug & - & rogue & - & - & - & - & - & - & - \\
\hline $\mathbf{j}$ & - & - & - & (yegg) & - & - & - & - & - & - & - & - & - & - & - & - \\
\hline z & - & (zig) & - & - & (zag) & - & - & - & - & - & - & - & - & - & - & - \\
\hline $\mathbf{s}$ & - & - & - & - & sag & - & - & sog & - & - & - & - & - & - & - & - \\
\hline $\int$ & - & - & - & - & shag & - & - & - & - & - & - & - & - & - & - & - \\
\hline $\mathbf{v}$ & - & - & vague & - & - & - & - & - & vogue & - & - & - & - & - & - & - \\
\hline f & - & fig & - & - & fag & - & fug & fog & - & - & - & - & - & - & fugue & - \\
\hline$h$ & - & - & (Hague) & - & hag & - & hug & hog & - & - & - & - & - & - & - & - \\
\hline$\delta$ & - & - & - & - & - & - & - & - & - & - & - & - & - & - & - & - \\
\hline$\theta$ & - & - & - & - & - & - & thug & - & - & $\cdots$ & - & - & - & - & - & - \\
\hline$w$ & - & wig & - & - & wag & - & - & - & - & - & - & - & - & - & - & - \\
\hline d3 & - & jig & - & - & jag & - & jug & jog & - & - & - & - & - & - & - & - \\
\hline ts & - & - & - & - & - & - & chug & - & - & - & - & - & - & - & - & - \\
\hline (ø) & - & - & - & egg & - & - & - & - & - & - & - & - & - & - & - & erg \\
\hline
\end{tabular}




\begin{tabular}{|c|c|c|c|c|c|c|c|c|c|c|c|c|c|c|c|c|}
\hline \multirow{2}{*}{$\begin{array}{c}\begin{array}{c}\text { First } \\
\text { Consonant }\end{array} \\
\end{array}$} & \multicolumn{16}{|c|}{ Vowel } \\
\hline & i & I & e & $\varepsilon$ & $æ$ & a & $\Lambda$ & 0 & 0 & $\mathrm{u}$ & $\mathbf{u}$ & al & II & au & ju & $3^{n}$ \\
\hline & \multicolumn{16}{|c|}{ Words Ending in p } \\
\hline$b$ & beep & - & - & - & - & (bop) & - & - & - & - & - & - & - & - & - & burp \\
\hline$d$ & deep & dip & - & $\cdots$ & - & - & - & - & dope & - & dupe & - & - & - & - & - \\
\hline g & - & - & gape & - & gap & - & - & - & - & - & (goop) & - & - & - & - & - \\
\hline$p$ & peep & pip & - & pep & pap & pop & pup & - & pope & - & (poop) & pipe & - & - & - & - \\
\hline$t$ & - & tip & tape & - & tap & top & - & - & tope & - & - & type & - & - & - & - \\
\hline k & keep & kip & cape & - & cap & cop & cup & - & cope & - & coop & - & - & - & - & - \\
\hline $\mathrm{m}$ & - & - & - & $\rightarrow$ & map & mop & - & - & mope & - & - & - & - & - & - & - \\
\hline $\mathrm{n}$ & - & nip & nape & - & nap & - & - & - & (nope) & - & - & - & - & - & - & - \\
\hline 1 & leap & lip & - & - & lap & lop & - & - & lope & - & loop & - & - & - & - & - \\
\hline $\mathrm{r}$ & reap & rip & rape & (rep) & rap & - & - & - & rope & - & - & ripe & - & - & - & - \\
\hline $\mathrm{j}$ & - & (yip) & - & (yep) & (уар) & - & (yup) & - & - & - & - & (yipe) & - & - & - & - \\
\hline$z$ & - & zip & - & - & zap & - & - & - & - & - & - & - & - & - & - & - \\
\hline s & seep & sip & - & - & sap & sop & sup & - & soap & - & soup & - & - & - & - & - \\
\hline $\int$ & sheep & ship & shape & - & - & shop & - & - & - & - & - & - & - & - & - & - \\
\hline$v$ & (VIP) & - & - & - & - & - & - & - & - & - & - & - & - & - & - & - \\
\hline f & - & - & - & - & - & fop & - & - & - & - & - & - & - & - & - & - \\
\hline h & heap & hip & - & (hep) & - & hop & - & - & hope & - & hoop & (hype) & - & - & - & - \\
\hline$\delta$ & - & - & - & - & - & - & - & - & - & - & - & - & - & - & - & - \\
\hline$\theta$ & - & - & - & - & - & - & - & - & - & - & - & - & - & - & - & - \\
\hline$w$ & weep & whip & - & $\cdots$ & - & wop & - & - & - & - & whoop & wipe & - & - & - & - \\
\hline ds & (Jeep) & gip & jape & - & (Jap) & - & - & - & - & - & - & - & - & - & - & - \\
\hline tJ & cheap & chip & chape & - & chap & chop & - & - & - & - & - & - & - & - & - & chirp \\
\hline (ש) & - & - & ape & $\cdots$ & - & fop) & up & - & - & - & - & - & - & - & - & - \\
\hline
\end{tabular}

\begin{tabular}{|c|c|c|c|c|c|c|c|c|c|c|c|c|c|c|c|c|}
\hline \multirow{2}{*}{$\begin{array}{c}\begin{array}{c}\text { First } \\
\text { Consonant }\end{array} \\
\end{array}$} & \multicolumn{16}{|c|}{ Vowel } \\
\hline & i & I & e & $\varepsilon$ & $æ$ & a & $\Lambda$ & 0 & $\underline{0}$ & $u$ & u & aI & II & $\mathrm{au}$ & ju & 3 \\
\hline & \multicolumn{16}{|c|}{ Words Ending in $t$} \\
\hline$b$ & beet & bit & bait & bet & bat & - & but & bought & boat & - & boot & bite & - & bout & - & (Burt) \\
\hline d & - & - & date & debt & - & dot & - & - & dote & - & - & - & - & doubt & - & $\operatorname{dirt}$ \\
\hline $\mathrm{g}$ & - & - & gate & get & gat & got & gut & ghat & goat & - & - & - & - & gout & - & girt \\
\hline$p$ & (Pete) & pit & pate & pet & pat & pot & putt & - & - & pout & - & - & - & pout & - & pert \\
\hline $\mathrm{t}$ & teat & (tit) & - & (Tet) & - & (tot) & (Tut) & taught & (tote) & - & toot & tight & - & tout & - & - \\
\hline$k$ & - & kit & (Kate) & - & cat & $\cot$ & cut & caught & coat & - & $\cos t$ & kite & - & - & cute & curt \\
\hline $\mathrm{m}$ & meat & mitt & mate & met & mat & - & mutt & - & moat & - & moot & might & - & - & mute & - \\
\hline $\mathbf{n}$ & neat & knit & - & net & gnat & not & nut & nought & note & - & newt & night & - & - & - & - \\
\hline 1 & - & lit & late & let & - & lot & - & - & - & - & loot & light & - & lout & - & - \\
\hline $\mathbf{r}$ & - & writ & rate & - & rat & rot & rut & wrought & wrote & - & root & right & - & route & - & - \\
\hline j & - & - & - & yet & - & yacht & - & - & - & - & - & - & - & - & - & - \\
\hline$z$ & - & (zit) & - & - & - & - & - & - & - & - & zoot & - & - & - & - & - \\
\hline s & seat & sit & sate & set & sat & sot & - & sought & - & soot & suit & sight & - & - & - & - \\
\hline J & sheet & (shit) & - & - & - & shot & shut & - & shoat & - & shoot & - & - & shout & - & shirt \\
\hline $\mathrm{v}$ & - & - & - & (vet) & vat & - & - & - & vote & - & - & - & - & - & - & - \\
\hline f & feet & fit & fate & - & fat & - & - & fought & - & foot & - & fight & - & - & - & - \\
\hline h & heat & hit & hate & - & hat & hot & hut & - & - & - & hoot & height & - & - & - & hurt \\
\hline 0 & - & - & - & - & that & & - & - & - & - & - & - & - & - & - & - \\
\hline$\theta$ & - & - & - & - & - & - & - & thought & - & - & - & - & - & - & - & - \\
\hline$w$ & wheat & wit & wait & wet & - & watt & what & - & - & - & - & white & - & - & - & - \\
\hline$d_{3}$ & - & - & - & jet & - & jot & jut & - & - & - & jute & - & - & - & - & - \\
\hline ts & cheat & chit & - & - & chat & - & - & - & - & - & - & - & - & - & - & - \\
\hline (b) & eat & it & ate & - & at & - & - & ought & oat & - & - & - & - & out & - & - \\
\hline
\end{tabular}




\begin{tabular}{|c|c|c|c|c|c|c|c|c|c|c|c|c|c|c|c|c|}
\hline \multirow{2}{*}{$\begin{array}{c}\begin{array}{c}\text { First } \\
\text { Consonant }\end{array} \\
\end{array}$} & \multicolumn{16}{|c|}{ Vowel } \\
\hline & $\mathrm{i}$ & $I$ & e & $\varepsilon$ & $\approx$ & a & $\Lambda$ & 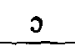 & o & $v$ & u & al & $\mathrm{si}$ & au & ju & 3 \\
\hline & \multicolumn{16}{|c|}{ Words Ending in $k$} \\
\hline b & beak & - & bake & beck & back & - & buck & balk & - & book & - & bike & - & - & - & - \\
\hline d & - & (Dick) & - & deck & - & dock & duck & - & - & - & duke & dike & - & - & - & dirk \\
\hline $\mathbf{g}$ & (geek) & - & - & - & - & - & - & gawk & - & (gook) & - & - & - & - & - & - \\
\hline $\mathbf{p}$ & peek & pick & - & peck & pack & pock & puck & - & poke & - & - & pike & - & - & puke & perk \\
\hline$t$ & teak & tick & take & (tech) & tack & tock & tuck & talk & (toke) & took & - & (tyke) & - & - & - & (Turk) \\
\hline k & - & kick & cake & - & - & cock & - & calk & (Coke) & cook & (kook) & $\rightarrow$ & - & - & - & (Kirk) \\
\hline $\mathrm{m}$ & meek & (mick) & make & - & (Mac) & mock & muck & - & - & - & - & (Mike) & - & - & - & murk \\
\hline $\mathbf{n}$ & - & nick & - & neck & knack & knock & - & - & - & nook & (nuke) & - & - & - & - & - \\
\hline 1 & leak & lick & lake & - & lack & lock & luck & - & - & look & (Luke) & like & - & - & - & lurk \\
\hline $\mathbf{r}$ & reek & (Rick) & rake & wreck & rack & rock & ruck & - & - & rook & - & - & - & - & - & - \\
\hline j & - & - & - & - & yak & - & (yuck) & - & yolk & - & - & - & - & - & - & - \\
\hline $\mathrm{z}$ & (Zeke) & - & - & - & $(\mathrm{Zac})$ & - & - & - & - & - & - & - & - & - & - & - \\
\hline$s$ & seek & sick & sake & - & sack & sock & suck & - & soak & - & - & (Psych) & - & - & - & - \\
\hline J & chic & - & shake & - & shack & shock & shuck & - & - & shook & - & - & - & - & - & shirk \\
\hline $\mathbf{v}$ & - & (Vic) & - & - & - & - & - & - & - & - & - & - & - & - & - & - \\
\hline f & - & - & fake & - & - & - & (fuck) & - & folk & - & - & - & - & - & - & - \\
\hline h & - & (hick) & hake & (heck) & (hack) & hock & (Huck) & hawk & - & hook & - & hike & - & - & - & - \\
\hline 0 & - & - & - & - & - & - & - & - & - & - & - & - & - & - & - & - \\
\hline$\theta$ & - & thick & - & - & - & - & - & - & - & - & - & - & - & - & - & - \\
\hline$w$ & week & wick & wake & - & whack & - & - & walk & woke & - & - & - & - & - & - & work \\
\hline$d_{3}$ & - & - & (Jake) & - & (Jack) & jock & - & - & joke & - & juke & - & - & - & - & jerk \\
\hline ts & cheek & chick & - & check & - & chock & chuck & chalk & choke & - & - & - & - & - & - & - \\
\hline (ø) & eke & - & ache & - & - & - & - & - & oak & - & - & - & - & - & - & irk \\
\hline
\end{tabular}

\begin{tabular}{|c|c|c|c|c|c|c|c|c|c|c|c|c|c|c|c|c|}
\hline \multirow{2}{*}{$\begin{array}{c}\text { First } \\
\text { Consonant }\end{array}$} & \multicolumn{16}{|c|}{ Vowel } \\
\hline & i & I & e & $\varepsilon$ & $\mathbf{a}$ & a & A & 0 & 0 & $\mathbf{u}$ & $\mathrm{u}$ & al & I & $\mathrm{au}$ & $\mathrm{ju}$ & 3 \\
\hline & \multicolumn{16}{|c|}{ Words Ending in $\mathrm{m}$} \\
\hline b & beam & - & - & - & - & bomb & bum & balm & - & - & boom & - & - & - & - & - \\
\hline d & deem & $\mathrm{dim}$ & dame & - & dam & (Dom) & dumb & - & dome & - & doom & dime & - & - & - & - \\
\hline g & - & - & game & - & gam & - & gum & - & - & - & - & - & - & - & - & - \\
\hline$p$ & - & - & - & - & (Pam) & - & - & palm & - & - & - & - & - & - & - & perm \\
\hline $\mathrm{t}$ & team & (Tim) & tame & - & - & (Tom) & - & - & tome & - & tomb & time & - & - & - & term \\
\hline k & - & (Kim) & came & - & cam & - & come & calm & comb & - & - & - & - & - & - & - \\
\hline $\mathrm{m}$ & - & - & maim & - & (mam) & (Mom) & mum & - & - & - & - & mime & - & - & - & - \\
\hline $\mathrm{n}$ & - & - & name & - & - & - & numb & - & gnome & - & - & - & - & - & - & - \\
\hline 1 & - & limb & lame & - & lamb & - & - & - & loam & - & loom & lime & - & - & - & - \\
\hline $\mathbf{r}$ & ream & $\mathrm{rim}$ & - & - & ram & (ROM) & rum & - & roam & - & room & thyme & - & - & - & - \\
\hline j & - & - & - & - & yam & - & (yum) & - & - & - & - & - & - & - & - & - \\
\hline$z$ & - & - & - & - & - & - & - & - & - & - & (zoom) & - & - & - & - & - \\
\hline $\mathbf{s}$ & seem & - & same & - & (Sam) & - & some & psalm & - & - & - & cyme & - & - & - & - \\
\hline s & - & shim & shame & - & sham & - & - & - & - & - & - & - & - & - & - & - \\
\hline$v$ & - & vim & - & - & - & - & - & - & - & - & (voom) & - & - & - & - & - \\
\hline $\mathrm{f}$ & - & - & fame & - & - & - & - & - & foam & - & - & - & - & - & fume & firm \\
\hline h & - & him & - & hem & ham & - & hum & - & home & - & whom & - & - & - & - & - \\
\hline$\delta$ & - & - & - & them & - & - & - & - & - & - & - & - & - & - & - & - \\
\hline$\theta$ & theme & - & - & - & - & - & thumb & - & - & - & - & - & - & - & - & therm \\
\hline $\mathbf{w}$ & - & whim & - & - & wham & - & - & - & - & - & womb & - & - & - & - & worm \\
\hline d3 & - & (Jim) & - & gem & jam & - & - & - & - & - & - & - & - & - & - & germ \\
\hline ts & - & - & - & - & - & - & chum & - & - & - & - & chime & - & - & - & - \\
\hline (b) & - & - & aim & $\mathbf{M}$ & am & - & (um) & - & ohm & - & - & $\left(l^{\prime} m\right)$ & - & - & - & - \\
\hline
\end{tabular}




\begin{tabular}{|c|c|c|c|c|c|c|c|c|c|c|c|c|c|c|c|c|}
\hline \multirow{2}{*}{$\begin{array}{c}\text { First } \\
\text { Consonant } \\
\end{array}$} & \multicolumn{16}{|c|}{ Vowel } \\
\hline & $\mathrm{i}$ & I & e & $\varepsilon$ & $\mathfrak{z}$ & a & $\Lambda$ & 0 & o & $u$ & $\mathbf{u}$ & aI & II & $\mathrm{aU}$ & ju & 3 \\
\hline & \multicolumn{16}{|c|}{ Words Ending in $\mathbf{n}$} \\
\hline b & bean & been & bane & (Ben) & ban & - & bun & - & bone & - & boon & - & - & - & - & burn \\
\hline d & dean & $\operatorname{din}$ & (Dane) & den & (Dan) & (Don) & done & dawn & - & - & dune & dine & - & down & - & (durn) \\
\hline g & - & - & gain & - & - & - & gun & - & - & - & goon & - & - & gown & - & - \\
\hline $\mathrm{p}$ & - & pin & pain & pen & pan & - & pun & pawn & pone & - & poon & pine & - & - & - & - \\
\hline$t$ & teen & $\operatorname{tin}$ & - & ten & $\tan$ & - & ton & - & tone & - & tune & tine & - & town & - & turn \\
\hline k & keen & kin & cane & Ken & can & (con) & - & - & cone & - & coon & kine & coin & - & - & kern \\
\hline $\mathrm{m}$ & mean & - & mane & men & $\operatorname{man}$ & - & - & - & moan & - & moon & mine & - & - & - & - \\
\hline $\mathrm{n}$ & - & - & - & - & - & - & none & - & known & - & noon & nine & - & - & - & - \\
\hline 1 & lean & - & lain & (Len) & - & - & - & lawn & loan & - & loon & line & loin & - & - & learn \\
\hline $\mathbf{r}$ & - & - & rain & wren & ran & (Ron) & run & - & roan & - & rune & (Rhine) & - & - & - & - \\
\hline $\mathrm{j}$ & yean & yin & - & yen & - & yon & - & yawn & - & - & - & - & - & - & - & yearn \\
\hline z & - & - & - & (zen) & - & - & - & - & zone & - & - & - & - & - & - & - \\
\hline $\mathbf{s}$ & seen & $\sin$ & sane & - & - & - & sun & - & sewn & - & soon & sign & - & - & - & - \\
\hline $\int$ & sheen & shin & (Shane) & - & - & - & shun & - & shone & - & - & shine & - & - & - & - \\
\hline $\mathrm{v}$ & - & - & vain & - & van & - & - & (Vaughn) & - & - & - & vine & - & - & - & - \\
\hline f & - & fin & feign & - & fan & - & fun & fawn & phone & - & - & fine & - & - & - & fern \\
\hline h & - & - & - & hen & - & - & (Hun) & - & hone & - & - & - & - & - & hewn & - \\
\hline d & - & - & - & then & than & - & - & - & - & - & - & (thine) & - & - & - & - \\
\hline$\theta$ & - & thin & thane & - & - & - & - & - & - & - & - & - & - & - & - & - \\
\hline $\mathbf{w}$ & wean & win & wane & when & - & wan & one & - & - & - & - & wine & - & - & - & - \\
\hline$d z$ & gene & gin & (Jane) & - & (Jan) & (John) & - & - & (Joan) & - & (June) & - & join & - & - & - \\
\hline if & - & chin & chais & - & - & - & - & - & - & - & - & chine & - & - & - & churn \\
\hline (6) & - & in & - & $\mathbf{N}$ & an & - & - & - & own & - & - & - & - & - & - & earn \\
\hline
\end{tabular}

\begin{tabular}{|c|c|c|c|c|c|c|c|c|c|c|c|c|c|c|c|c|}
\hline \multirow{2}{*}{$\begin{array}{c}\text { First } \\
\text { Consonant }\end{array}$} & \multicolumn{16}{|c|}{ Vowel } \\
\hline & $\mathrm{i}$ & I & $\mathbf{e}$ & $\varepsilon$ & $æ$ & $\mathbf{a}$ & $\Lambda$ & 5 & 0 & $\boldsymbol{u}$ & $\mathbf{u}$ & al & II & au & ju & $x^{x}$ \\
\hline & \multicolumn{16}{|c|}{ Words Ending in I } \\
\hline b & - & bing & - & - & bang & - & bung & bong & - & - & - & - & - & - & - & - \\
\hline d & - & ding & - & - & (dang) & - & dung & dong & - & - & - & - & - & - & - & - \\
\hline $\mathbf{g}$ & - & - & - & - & gang & - & - & gong & - & - & - & - & - & - & - & - \\
\hline $\mathbf{p}$ & - & ping & - & - & pang & - & - & pong & - & - & - & - & - & - & - & - \\
\hline$t$ & - & ting & - & - & tang & - & tongue & tong & - & - & - & - & - & - & - & - \\
\hline $\mathbf{k}$ & - & ling & - & - & - & - & - & (Kong) & - & - & - & - & - & - & - & - \\
\hline m & - & (Ming) & - & - & - & - & mung & - & - & - & - & - & - & - & - & - \\
\hline $\mathrm{n}$ & - & - & - & - & - & - & - & - & - & - & - & - & - & - & - & - \\
\hline 1 & - & - & - & - & - & - & lung & long & - & - & - & - & - & - & - & - \\
\hline $\mathbf{r}$ & - & ring & - & - & rang & - & rung & wrong & - & - & - & - & - & - & - & - \\
\hline $\mathbf{j}$ & - & - & - & - & yang & - & young & - & - & (Jung) & - & - & - & - & - & - \\
\hline z & - & (zing) & - & - & - & - & - & - & - & - & - & - & - & - & - & - \\
\hline $\mathbf{s}$ & - & sing & - & - & sang & - & sung & song & - & - & - & - & - & - & - & - \\
\hline J & - & - & - & - & - & - & - & - & - & - & - & - & - & - & - & - \\
\hline $\mathrm{v}$ & - & - & - & - & vang & - & - & - & - & - & - & - & - & - & - & - \\
\hline f & - & - & - & - & fang & - & - & - & - & - & - & - & - & - & - & - \\
\hline h & - & - & - & - & hang & - & hung & - & - & - & - & - & - & - & - & - \\
\hline$\delta$ & - & - & - & - & - & - & - & - & - & - & - & - & - & - & - & - \\
\hline$\theta$ & - & thing & - & - & - & - & - & thong & - & - & - & - & - & - & - & - \\
\hline $\mathbf{w}$ & - & wing & - & - & (Wang) & - & - & - & - & - & - & - & - & - & - & - \\
\hline d3 & - & - & - & - & - & - & - & - & - & - & - & - & - & - & - & - \\
\hline ts & - & - & - & - & - & - & - & - & - & - & - & - & - & - & - & - \\
\hline (a) & - & - & - & - & - & - & - & - & - & - & - & - & - & - & - & - \\
\hline
\end{tabular}




\begin{tabular}{|c|c|c|c|c|c|c|c|c|c|c|c|c|c|c|c|c|}
\hline \multirow{2}{*}{$\begin{array}{c}\begin{array}{c}\text { First } \\
\text { Consonant }\end{array} \\
\end{array}$} & \multicolumn{16}{|c|}{ Vowel } \\
\hline & $\mathrm{i}$ & I & $\mathrm{e}$ & $\varepsilon$ & $\boldsymbol{x}$ & a & $\Lambda$ & 5 & 0 & $u$ & $\mathbf{u}$ & aI & II & au & ju & 3 \\
\hline & \multicolumn{16}{|c|}{ Words Ending in 1} \\
\hline b & - & bill & bail & bell & - & - & - & ball & bowl & bull & - & bile & boil & bowel & - & burl \\
\hline d & deal & dill & dale & dell & - & doll & dull & - & dole & - & - & dial & - & dowel & - & - \\
\hline g & - & gill & gale & - & gal & - & gull & gall & goal & - & ghoul & guile & - & - & - & girl \\
\hline p & peel & pill & pail & (pell) & pal & - & - & (Paul) & pole & pull & pool & pile & - & - & - & pearl \\
\hline$t$ & teal & till & tail & tell & - & - & - & tall & toll & - & tool & tile & toil & towel & - & - \\
\hline $\mathbf{k}$ & keel & kill & kale & - & - & - & cull & call & coal & - & cool & chyle & coil & cowl & - & curl \\
\hline $\mathrm{m}$ & meal & mill & mail & (mell) & - & (moll) & mull & mall & mole & - & - & mile & moil & - & mule & (Merle) \\
\hline $\mathbf{n}$ & (Neal) & nil & nail & (Nell) & - & - & null & - & knoll & - & - & (Nile) & - & - & - & - \\
\hline 1 & - & (Lil) & - & - & - & loll & lull & - & - & - & - & - & loyal & - & - & - \\
\hline$r$ & real & rill & rail & - & - & - & - & - & role & - & rule & rile & roil & - & - & - \\
\hline $\mathrm{j}$ & - & - & (Yale) & yell & - & - & - & yawl & - & - & (Yule) & - & - & - & - & - \\
\hline$z$ & zeal & - & - & - & - & - & - & - & - & - & - & - & - & - & - & - \\
\hline $\mathbf{s}$ & seal & sill & sail & sell & (Sal) & - & - & (Sol) & sole & - & - & - & soil & - & - & - \\
\hline $\int$ & (she'll) & (shill) & shale & shell & shall & - & - & shaw! & shoal & - & - & - & - & - & - & (Shirl) \\
\hline$v$ & veal & - & veil & - & (Val) & - & - & - & vole & - & - & vile & - & vowel & - & - \\
\hline $\mathrm{f}$ & feel & fill & fail & fell & - & - & - & fall & foal & full & fool & file & foil & foul & fuel & furl \\
\hline h & heal & hill & hail & hell & (Hal) & - & hull & hall & hole & - & (who'll) & (heil) & (Hoyle) & howl & - & hurl \\
\hline 0 & - & - & - & - & - & - & - & - & - & - & - & - & - & - & - & - \\
\hline$\theta$ & - & thill & - & - & - & - & - & - & - & - & - & - & - & - & - & - \\
\hline$w$ & wheel & will & whale & well & - & - & - & wall & - & wool & - & while & - & - & - & whirl \\
\hline d3 & - & (Jill) & jail & gel & - & - & - & - & - & - & jewel & - & - & jowl & - & - \\
\hline if & - & chill & - & - & - & - & - & - & - & - & - & - & - & - & - & churl \\
\hline (б) & ex & ill & ale & L & $(\mathrm{Al})$ & - & - & all & - & - & - & aisle & oil & owl & you'll & (Earl) \\
\hline
\end{tabular}

\begin{tabular}{|c|c|c|c|c|c|c|c|c|c|c|c|c|c|c|c|c|}
\hline \multirow{2}{*}{$\begin{array}{c}\text { First } \\
\text { Consonant }\end{array}$} & \multicolumn{16}{|c|}{ Vowel } \\
\hline & $\mathrm{i}$ & I & e & $\varepsilon$ & $\mathfrak{x}$ & a & $\Lambda$ & $\mathrm{s}$ & 0 & $U$ & $\mathbf{u}$ & al & OI & au & ju & 3 \\
\hline
\end{tabular}

Words Ending in $\mathbf{r}$

(Monosyllabicity of the words with diphthongs, e.g., buyer, bower, foyer, is debatable.)

\begin{tabular}{|c|c|c|c|c|c|c|c|c|c|c|c|c|c|c|c|c|}
\hline b & beer & - & bear & - & - & bar & - & bore & - & - & - & buyer & - & bower & - & - \\
\hline $\mathrm{d}$ & deer & - & dare & - & - & - & - & door & - & - & - & dire & - & dour & - & - \\
\hline g & gear & - & - & - & - & - & - & gore & - & - & - & - & - & - & - & - \\
\hline p & peer & - & pair & - & - & par & - & pore & - & - & 一 & pyre & - & power & pure & - \\
\hline$t$ & tear & - & tear & - & - & $\operatorname{tar}$ & - & tore & - & - & - & tire & - & tower & - & - \\
\hline $\mathbf{k}$ & - & - & care & - & - & car & - & core & - & - & 一 & - & - & cower & cure & - \\
\hline $\mathrm{m}$ & mere & - & mare & - & - & $\operatorname{mar}$ & - & more & - & - & moor & mire & - & - & - & - \\
\hline $\mathrm{n}$ & near & - & - & - & - & - & - & nor & - & - & - & - & - & - & 一 & - \\
\hline 1 & leer & - & lair & - & - & - & - & lore & - & - & lure & lyre & - & - & - & - \\
\hline $\mathbf{r}$ & rear & - & rare & - & - & - & - & roar & - & - & - & - & - & - & - & - \\
\hline j & year & - & - & - & - & - & - & your & - & - & - & - & - & - & - & - \\
\hline$z$ & - & - & - & - & - & Czar & - & - & - & - & - & - & - & - & - & - \\
\hline $\mathbf{s}$ & sear & - & - & - & - & - & - & sore & - & - & - & sire & - & sour & - & - \\
\hline f & shear & - & share & - & - & - & - & shore & - & - & - & - & - & shower & - & - \\
\hline$v$ & veer & - & - & - & - & - & - & - & - & - & - & - & - & - & - & - \\
\hline f & fear & - & fare & - & - & far & - & for & - & - & - & fire & foyer & - & - & - \\
\hline $\mathbf{h}$ & hear & - & hair & - & - & - & - & whore & - & - & - & hire & - & (how're) & - & - \\
\hline 0 & - & - & there & - & - & - & - & - & - & - & - & - & - & - & - & - \\
\hline$\theta$ & - & - & - & - & - & - & - & (Thor) & - & - & - & - & - & - & - & - \\
\hline$w$ & (we're) & - & wear & - & - & - & - & war & - & - & - & wire & - & - & - & - \\
\hline d3 & jeer & - & - & - & - & jar & - & - & - & - & - & gyre & - & - & - & - \\
\hline t & cheer & - & chair & - & - & char & - & chore & - & - & - & - & - & - & - & - \\
\hline (ø) & ear & - & heir & - & - & are & - & or & - & - & - & ire & - & our & - & - \\
\hline
\end{tabular}




\begin{tabular}{|c|c|c|c|c|c|c|c|c|c|c|c|c|c|c|c|c|}
\hline \multirow{2}{*}{$\begin{array}{c}\text { First } \\
\text { Consonant }\end{array}$} & \multicolumn{16}{|c|}{ Vowel } \\
\hline & $\mathrm{i}$ & $I$ & $\mathrm{e}$ & $\varepsilon$ & $æ$ & $\mathbf{a}$ & $\Lambda$ & 0 & 0 & $u$ & $\mathbf{u}$ & al & II & au & ju & 3 \\
\hline & \multicolumn{16}{|c|}{ Words Ending in $\mathrm{z}$} \\
\hline$b$ & bees & (biz) & bays & - & - & - & buzz & - & bows & - & booze & buys & boys & boughs & - & burrs \\
\hline d & (D's) & - & days & - & - & - & does & - & doze & - & dues & dies & - & - & - & - \\
\hline g & - & - & gays & - & - & - & - & gauze & goes & - & - & guys & - & - & - & - \\
\hline $\mathrm{p}$ & peas & - & pays & - & - & - & - & paws & pose & - & - & pies & poise & - & pews & purrs \\
\hline$t$ & tease & 'tis & - & - & - & - & - & taws & toes & - & (two's) & ties & toys & - & - & - \\
\hline $\mathrm{k}$ & keys & - & cays & - & - & - & (cuz) & cause & - & - & $\operatorname{coos}$ & - & - & cows & - & curs \\
\hline $\mathrm{m}$ & - & (Ms.) & maze & - & - & - & - & - & mows & - & moos & - & - & - & muse & - \\
\hline $\mathrm{n}$ & knees & - & neighs & - & - & - & - & gnaws & nose & - & news & - & noise & - & - & - \\
\hline 1 & lees & $(\mathrm{Liz})$ & lays & - & - & - & - & laws & lows & - & lose & lies & - & - & - & - \\
\hline$r$ & - & - & rays & - & $(\operatorname{razz})$ & - & - & - & rose & - & ruse & rise & - & - & - & - \\
\hline $\mathrm{j}$ & - & - & - & - & - & - & - & yaws & - & - & use & - & - & - & - & - \\
\hline z & - & - & - & - & - & - & - & - & - & - & - & - & - & - & - & - \\
\hline $\mathrm{s}$ & sieze & - & - & says & - & - & - & saws & sows & - & sues & size & - & sows & - & sirs \\
\hline $\int$ & (she's) & - & - & - & - & Shah's & - & - & shows & - & shoes & shies & - & - & - & - \\
\hline $\mathrm{v}$ & - & - & vase & - & - & vase & - & - & - & - & - & - & - & vows & views & - \\
\hline f & fees & fizz & faze & fez & - & - & fuzz & - & foes & - & - & - & - & - & fuse & furs \\
\hline h & (he's) & his & haze & - & has & - & - & haws & hose & - & whose & - & - & house & hues & hers \\
\hline 0 & these & - & - & - & - & - & - & - & those & - & - & - & - & - & - & - \\
\hline$\theta$ & - & - & - & - & - & - & - & thaws & - & - & - & thighs & - & - & - & - \\
\hline $\mathbf{w}$ & wheeze & - & ways & - & - & - & was & - & woes & - & woos & wise & - & (wows) & - & whirs \\
\hline d3 & - & - & - & - & jazz & - & - & jaws & (Joe's) & - & (Jews) & - & joys & - & - & - \\
\hline t & cheese & - & - & - & - & - & - & - & chose & - & choose & - & - & - & - & - \\
\hline (d) & ease & is & A's & - & as & $(\mathrm{Oz})$ & - & awes & owes & - & ooze & eyes & - & - & use & - \\
\hline
\end{tabular}

\begin{tabular}{|c|c|c|c|c|c|c|c|c|c|c|c|c|c|c|c|c|}
\hline \multirow{2}{*}{$\begin{array}{c}\text { First } \\
\text { Consonant }\end{array}$} & \multicolumn{16}{|c|}{ Vowel } \\
\hline & $\mathrm{i}$ & I & e & $\varepsilon$ & $\dddot{\gtrless}$ & $\mathbf{a}$ & $\Lambda$ & $\mathbf{0}$ & $\mathbf{o}$ & $u$ & $\mathbf{u}$ & al & II & au & ju & $3^{2}$ \\
\hline & \multicolumn{16}{|c|}{ Words Ending in s } \\
\hline b & - & - & base & (Bess) & bass & - & bus & boss & - & - & - & - & - & - & - & - \\
\hline d & - & - & dace & - & - & - & - & - & dose & - & deuce & dice & - & douse & - & - \\
\hline $\mathrm{g}$ & geese & - & - & guess & gas & - & (Gus) & - & - & - & goose & - & - & gauss & - & - \\
\hline$p$ & piece & (piss) & pace & - & pass & - & pus & - & - & (puss) & - & - & - & - & puce & purse \\
\hline$t$ & - & - & 一 & (Tess) & (Tass) & - & - & toss & - & - & - & - & - & - & - & terse \\
\hline k & - & kiss & case & - & - & - & (cuss) & $\cos$ & - & - & - & - & - & - & - & curse \\
\hline $\mathrm{m}$ & - & miss & mace & mess & mass & - & muss & moss & - & - & moose & mice & - & mouse & - & - \\
\hline $\mathrm{n}$ & niece & - & - & - & - & - & - & - & - & - & noose & nice & - & - & - & nurse \\
\hline 1 & lease & - & lace & less & lass & - & - & loss & - & - & loose & lice & - & louse & - & - \\
\hline $\mathbf{r}$ & - & - & race & - & - & - & (Russ) & - & - & - & - & rice & - & - & - & - \\
\hline $\mathrm{j}$ & - & - & - & yes & - & - & - & - & - & - & use & - & - & - & - & - \\
\hline$z$ & & - & - & - & - & - & - & - & - & - & (Zeus) & - & - & - & - & - \\
\hline s & cease & (Sis) & - & cess & (sass) & - & - & sauce & - & - & - & - & - & souse & - & - \\
\hline f & - & - & - & - & - & - & - & - & - & - & - & - & - & - & - & - \\
\hline$v$ & - & - & - & - & - & - & - & - & - & - & - & vice & voice & - & - & verse \\
\hline $\mathrm{f}$ & - & - & face & - & - & fosse & fuss & fosse & - & - & - & - & - & - & - & - \\
\hline h & - & hiss & - & - & - & - & - & - & - & - & - & - & - & house & - & hearse \\
\hline 0 & - & this & - & - & - & - & thus & - & - & - & - & - & - & - & - & - \\
\hline$\theta$ & - & - & - & - & - & - & thus & - & - & - & - & - & - & - & - & - \\
\hline $\mathbf{w}$ & - & - & - & - & - & - & - & - & - & - & - & - & - & - & - & worse \\
\hline d3 & - & - & - & - & - & joss & - & - & - & - & juice & - & (Joyce) & - & - & - \\
\hline ts & - & - & chase & chess & - & - & - & - & - & - & - & - & choice & - & - & - \\
\hline (ø) & - & - & ace & $S$ & ass & os & us & - & os & - & - & ice & - & - & use & - \\
\hline
\end{tabular}




\begin{tabular}{|c|c|c|c|c|c|c|c|c|c|c|c|c|c|c|c|c|}
\hline \multirow{2}{*}{$\begin{array}{c}\text { First } \\
\text { Consonant } \\
\end{array}$} & \multicolumn{16}{|c|}{ Vowel } \\
\hline & $i$ & $I$ & e & $\varepsilon$ & $\approx$ & a & $\Lambda$ & 0 & 0 & $v$ & $\mathrm{u}$ & aI & II & au & $\mathrm{ju}$ & 3 \\
\hline & \multicolumn{16}{|c|}{ Words Ending in $\int$} \\
\hline b & - & - & - & - & bash & - & - & - & - & bush & - & - & - & - & - & - \\
\hline d & - & dish & - & - & dash & - & - & - & - & - & douche & - & - & - & - & - \\
\hline g & - & - & - & - & gash & (gosh) & gush & - & gauche & - & - & - & - & - & - & - \\
\hline p & - & (pish) & - & - & - & (posh) & - & - & - & push & - & - & - & - & - & - \\
\hline$t$ & - & - & - & - & - & - & - & - & - & - & - & - & - & - & - & - \\
\hline$k$ & quiche & - & - & - & cash & - & - & - & - & - & - & - & - & - & - & - \\
\hline $\mathbf{m}$ & - & - & - & mesh & mash & - & mush & - & - & - & - & - & - & - & - & - \\
\hline $\mathbf{n}$ & - & - & - & - & gnash & (nosh) & - & - & - & - & - & - & - & - & - & - \\
\hline 1 & leash & - & - & - & lash & - & lush & - & - & - & - & - & - & - & - & - \\
\hline$r$ & - & - & - & - & rash & - & rush & - & - & - & - & - & - & - & - & - \\
\hline $\mathbf{j}$ & - & - & - & - & - & - & - & - & - & - & - & - & - & - & - & - \\
\hline$z$ & - & - & - & - & - & - & - & - & - & - & - & - & - & - & - & - \\
\hline s & - & - & - & - & sash & - & - & - & - & - & - & - & - & - & - & - \\
\hline $\int$ & - & - & - & - & - & - & - & - & - & - & - & - & - & - & - & - \\
\hline $\mathbf{v}$ & - & - & - & - & - & - & - & - & - & - & - & - & - & - & - & - \\
\hline f & fiche & fish & - & - & - & - & - & - & - & - & - & - & - & - & - & - \\
\hline h & - & - & - & - & hash & - & hush & - & - & - & - & - & - & - & - & - \\
\hline o & - & - & - & - & - & - & - & - & - & - & - & - & - & - & - & - \\
\hline$\theta$ & - & - & - & - & - & - & - & - & - & - & - & - & - & - & - & - \\
\hline $\mathbf{w}$ & - & wish & - & - & - & wash & - & - & - & (whoosh) & - & - & - & - & - & - \\
\hline d3 & - & - & - & - & - & (josh) & - & - & - & - & - & - & - & - & - & - \\
\hline t) & - & - & - & - & - & - & - & - & - & - & - & - & - & - & - & - \\
\hline (פ) & - & - & - & - & ash & - & - & - & - & - & - & - & - & - & - & - \\
\hline
\end{tabular}

\begin{tabular}{|c|c|c|c|c|c|c|c|c|c|c|c|c|c|c|c|c|}
\hline \multirow{2}{*}{$\begin{array}{c}\text { First } \\
\text { Consonant }\end{array}$} & \multicolumn{16}{|c|}{ Vowel } \\
\hline & i & I & e & $\varepsilon$ & $æ$ & $\mathbf{a}$ & $\boldsymbol{\Lambda}$ & 0 & 0 & $v$ & $\mathbf{1}$ & $\mathbf{a I}$ & $I$ & av & ju & $3^{*}$ \\
\hline
\end{tabular}

\section{Words Ending in v}

\begin{tabular}{|c|c|c|c|c|c|c|c|c|c|c|c|c|c|c|c|c|}
\hline b & - & - & - & - & - & - & - & - & - & - & - & - & - & - & - & - \\
\hline d & - & - & (Dave) & - & - & - & dove & - & dove & - & - & dive & - & - & - & - \\
\hline g & - & give & gave & - & - & - & - & - & - & - & - & - & - & - & - & - \\
\hline$p$ & peeve & - & pave & - & - & - & - & - & - & - & - & - & - & - & - & - \\
\hline$t$ & - & - & - & - & - & - & - & - & - & - & - & - & - & - & - & - \\
\hline k & - & - & cave & - & - & - & - & - & cove & - & - & - & - & - & - & curve \\
\hline m & - & - & - & - & - & - & - & - & mauve & - & move & - & - & - & - & (Merv) \\
\hline $\mathbf{n}$ & - & - & knave & - & - & - & - & - & - & - & - & - & - & - & - & nerve \\
\hline I & leave & live & lave & - & - & - & love & - & - & - & - & live & - & - & - & - \\
\hline $\mathbf{r}$ & reeve & - & rave & - & - & - & - & - & rove & - & - & - & - & - & - & - \\
\hline $\mathbf{j}$ & - & - & - & - & - & - & - & - & - & - & (you've) & - & - & - & - & - \\
\hline $\mathbf{z}$ & - & - & - & - & - & - & - & - & - & - & - & - & - & - & - & - \\
\hline $\mathbf{s}$ & - & sieve & save & - & - & - & - & - & - & - & - & - & - & - & - & serve \\
\hline $\int$ & sheave & - & shave & - & - & - & shove & - & - & - & - & - & - & - & - & - \\
\hline$v$ & - & - & - & - & - & -- & - & - & - & - & - & - & - & - & - & verve \\
\hline f & - & - & - & - & - & - & - & - & - & - & - & five & - & - & - & - \\
\hline $\mathbf{h}$ & heave & - & - & - & have & - & - & - & hove & - & - & hive & - & - & - & - \\
\hline ס & - & - & (they've) & - & - & - & - & - & - & - & - & - & - & - & - & - \\
\hline$\theta$ & thieve & - & - & - & - & - & - & - & - & - & - & - & - & - & - & - \\
\hline $\mathbf{w}$ & weave & - & wave & - & - & - & - & - & wove & - & - & - & - & - & - & - \\
\hline d3 & - & - & - & - & - & - & - & - & (Jove) & - & - & jive & - & - & - & - \\
\hline ts & - & - & - & - & - & - & - & - & - & - & - & - & - & - & - & - \\
\hline (6) & (Eve) & - & - & - & - & - & of & - & - & - & - & (I've) & - & - & (you've) & (Irv) \\
\hline
\end{tabular}




\begin{tabular}{|c|c|c|c|c|c|c|c|c|c|c|c|c|c|c|c|c|}
\hline \multirow{2}{*}{$\begin{array}{c}\text { First } \\
\text { Consonant } \\
\end{array}$} & \multicolumn{16}{|c|}{ Vowel } \\
\hline & $\mathrm{i}$ & $\mathrm{I}$ & $\mathrm{e}$ & $\varepsilon$ & $\mathfrak{x}$ & $\mathbf{a}$ & $\Lambda$ & 0 & 0 & $u$ & $\mathbf{u}$ & aI & II & au & ju & 3 \\
\hline & \multicolumn{16}{|c|}{ Words Ending in $\mathbf{f}$} \\
\hline b & beef & (Biff) & - & - & - & - & buff & - & - & - & - & - & - & - & - & - \\
\hline d & - & - & - & deaf & - & - & (duff) & doff & - & - & - & - & - & - & - & - \\
\hline $\mathbf{g}$ & - & - & - & - & gaffe & - & guff & - & - & - & goof & - & - & - & - & - \\
\hline $\mathrm{p}$ & - & - & - & - & - & - & puff & - & - & (poof) & (poof) & - & - & - & - & - \\
\hline $\mathrm{t}$ & - & tiff & - & - & - & - & tough & toff & - & - & - & - & - & - & - & turf \\
\hline k & - & - & - & - & calf & - & cuff & cough & - & - & - & - & - & - & - & - \\
\hline $\mathrm{m}$ & - & miff & - & - & - & - & muff & - & - & - & - & - & - & - & - & - \\
\hline $\mathrm{n}$ & - & - & - & - & - & - & - & - & - & - & - & knife & - & - & - & (nerf) \\
\hline 1 & leaf & - & - & - & laugh & - & luff & - & loaf & - & - & life & - & - & - & - \\
\hline$r$ & reef & riff & - & - & - & - & rough & - & - & - & roof & rife & - & - & - & - \\
\hline $\mathbf{j}$ & - & - & - & - & - & - & - & - & - & - & - & - & - & - & - & - \\
\hline$z$ & - & - & - & - & - & - & - & - & - & - & - & - & - & - & - & - \\
\hline $\mathrm{s}$ & - & - & - & - & - & - & - & - & - & - & - & - & - & - & - & surf \\
\hline J & sheaf & - & - & chef & - & - & - & - & - & - & - & - & - & - & - & - \\
\hline $\mathrm{v}$ & - & - & - & - & - & - & - & - & - & - & - & - & - & - & - & - \\
\hline $\mathrm{f}$ & fief & - & - & - & - & - & - & - & - & - & - & fife & - & - & - & - \\
\hline h & - & - & - & - & half & - & huff & - & - & hoof & - & - & - & - & - & - \\
\hline$\delta$ & - & - & - & - & - & - & - & - & - & - & - & - & - & - & - & - \\
\hline$\theta$ & thief & - & - & - & - & - & - & - & - & - & - & - & - & - & - & - \\
\hline w & - & whiff & waif & - & - & - & - & - & - & (woof) & - & wife & - & - & - & - \\
\hline dz & - & (jiff) & - & (Jeff) & - & - & - & - & - & - & - & - & - & - & - & - \\
\hline ts & chief & - & chafe & - & chaff & - & - & - & - & - & - & - & - & - & - & - \\
\hline (b) & - & if & - & $F$ & - & - & - & off & af & - & (oof) & - & - & - & - & - \\
\hline
\end{tabular}

\begin{tabular}{|c|c|c|c|c|c|c|c|c|c|c|c|c|c|c|c|c|}
\hline \multirow{2}{*}{$\begin{array}{c}\text { First } \\
\text { Consonant }\end{array}$} & \multicolumn{16}{|c|}{ Vowel } \\
\hline & $\mathrm{i}$ & I & e & $\varepsilon$ & $\mathbf{z}$ & $\mathbf{a}$ & $\Lambda$ & 0 & 0 & $v$ & $\mathbf{u}$ & al & II & au & $\mathrm{ju}$ & $3^{5}$ \\
\hline
\end{tabular}

\section{Words Ending in $\delta$ or $\theta$}

(Words with $\delta$ marked with an asterisk.)

\begin{tabular}{|c|c|c|c|c|c|c|c|c|c|c|c|c|c|c|c|c|}
\hline b & - & - & *bathe & (Beth) & bath & - & - & - & both & - & booth & - & - & - & - & birth \\
\hline d & - & - & - & death & - & - & - & - & - & - & - & - & - & - & - & dearth \\
\hline g & - & - & - & - & - & - & - & - & - & - & - & - & - & - & - & girth \\
\hline $\mathbf{p}$ & - & pith & - & - & path & - & - & - & - & - & - & - & - & - & - & Perth \\
\hline $\mathrm{t}$ & loeth/"teethe & - & - & - & - & - & - & - & - & - & tooth & "ithe & - & - & - & - \\
\hline k & (Keith) & kith & - & - & - & - & - & - & - & - & couth & - & - & - & - & - \\
\hline m & - & myth & - & - & math & - & - & moth & - & - & - & - & - & mouth $/{ }^{*}$ mouth & - & mirth \\
\hline $\mathbf{n}$ & - & - & - & - & - & - & - & - & - & - & - & - & - & - & - & - \\
\hline 1 & - & - & "lathe & - & lath & - & - & - & - & - & - & *lithe & - & - & - & - \\
\hline$r$ & wreath & - & wraith & - & wrath & - & - & - & - & - & (Ruth) & - & - & - & - & - \\
\hline $\mathrm{j}$ & - & - & - & - & - & - & - & - & - & - & youth & - & - & - & - & - \\
\hline $\mathrm{z}$ & & - & - & - & - & - & - & - & - & - & - & - & - & - & - & - \\
\hline $\mathbf{s}$ & *secthe & - & - & (Seth) & - & - & - & - & - & - & *soothe & - & - & south & - & - \\
\hline J & sheath & - & - & - & - & - & - & - & - & - & - & - & - & - & - & - \\
\hline$v$ & - & - & - & - & - & - & - & - & - & - & - & - & - & - & - & - \\
\hline f & - & - & faith & - & - & - & - & - & - & - & - & - & - & - & - & - \\
\hline h & - & - & - & - & hath & - & - & - & - & - & - & - & - & - & - & - \\
\hline 0 & - & - & - & - & - & - & - & - & - & - & - & - & - & - & - & - \\
\hline$\theta$ & - & - & - & - & - & - & - & - & - & - & - & - & - & - & - & - \\
\hline w & - & with & - & - & - & - & - & - & - & - & - & - & - & - & - & worth \\
\hline$d_{3}$ & - & - & - & - & - & - & - & - & - & - & - & - & - & - & - & - \\
\hline if & - & - & - & - & - & - & - & - & - & - & - & - & - & - & - & - \\
\hline (छ) & - & - & - & - & - & - & - & - & oath & - & - & - & - & - & youth & earth \\
\hline
\end{tabular}




\begin{tabular}{|c|c|c|c|c|c|c|c|c|c|c|c|c|c|c|c|c|}
\hline \multirow{2}{*}{$\begin{array}{c}\text { First } \\
\text { Consonant }\end{array}$} & \multicolumn{16}{|c|}{ Vowel } \\
\hline & $\mathrm{i}$ & I & $\mathrm{e}$ & $\varepsilon$ & $æ$ & a & $\Lambda$ & $\boldsymbol{0}$ & 0 & $u$ & $\mathrm{u}$ & aI & $\boldsymbol{I}$ & $a U$ & ju & 3 \\
\hline & \multicolumn{16}{|c|}{$\begin{array}{l}\text { Words Ending in d } 3 \text { or } 3 \\
\text { (Words with } 3 \text { marked with an asterisk.) }\end{array}$} \\
\hline b & - & - & *beige & - & badge & - & budge & - & - & - & - & - & - & - & - & - \\
\hline d & - & - & - & - & - & dodge & - & - & doge & - & - & - & - & - & - & dirge \\
\hline $\mathbf{g}$ & - & - & gauge & - & - & - & - & - & - & - & - & - & - & gouge & - & - \\
\hline $\mathbf{p}$ & - & - & page & - & - & - & - & - & - & - & - & - & - & - & - & purge \\
\hline $\mathrm{t}$ & - & - & - & - & - & - & - & - & - & - & - & - & - & - & - & - \\
\hline k & - & - & cage & kedge & cadge & - & - & - & - & - & - & - & - & - & - & - \\
\hline $\mathrm{m}$ & - & (Midge) & - & - & - & - & - & - & - & - & - & - & - & - & - & merge \\
\hline $\mathrm{n}$ & - & - & - & - & - & - & nudge & - & - & (nudge) & - & - & - & - & - & - \\
\hline I & liege & - & - & ledge & - & lodge & - & - & - & - & - & - & - & - & - & - \\
\hline$r$ & - & ridge & rage & (Reg) & - & - & - & - & - & - & $*_{\text {rouge }}$ & - & - & - & - & - \\
\hline $\mathrm{j}$ & - & - & - & - & - & - & - & - & - & - & - & - & - & - & - & - \\
\hline$z$ & - & - & - & - & - & - & - & - & - & - & - & - & - & - & - & - \\
\hline$s$ & siege & - & sage & sedge & - & - & - & - & - & - & - & - & - & - & - & surge \\
\hline J & - & - & - & - & - & - & - & - & - & - & - & - & - & - & - & - \\
\hline v & - & - & - & - & - & - & - & - & - & - & - & - & - & - & - & verge \\
\hline f & - & - & - & - & - & - & fudge & - & - & - & - & - & - & - & - & - \\
\hline h & - & - & - & hedge & hajj & - & - & - & - & - & - & - & - & - & huge & - \\
\hline ठ & - & - & - & - & - & - & - & - & - & - & - & - & - & - & - & - \\
\hline$\theta$ & - & - & - & - & - & - & - & - & - & - & - & - & - & - & - & - \\
\hline w & - & - & wage & wedge & - & - & - & - & - & - & - & - & - & - & - & - \\
\hline dz & - & - & - & - & - & - & judge & - & - & - & - & - & - & - & - & - \\
\hline ts & - & - & - & - & - & - & - & - & - & - & - & - & - & - & - & - \\
\hline (פ) & - & - & age & edge & - & - & - & - & - & - & - & - & - & - & - & urge \\
\hline
\end{tabular}

\begin{tabular}{|c|c|c|c|c|c|c|c|c|c|c|c|c|c|c|c|c|}
\hline \multirow{2}{*}{$\begin{array}{c}\begin{array}{c}\text { First } \\
\text { Consonant }\end{array} \\
\end{array}$} & \multicolumn{16}{|c|}{ Vowel } \\
\hline & $\mathrm{i}$ & I & e & $\varepsilon$ & $\mathfrak{x}$ & a & $\Lambda$ & 0 & 0 & $u$ & u & aI & วI & au & ju & 3 \\
\hline & \multicolumn{16}{|c|}{ Words Ending in $\mathbf{t} \int$} \\
\hline b & beach & bitch & - & - & batch & botch & - & - & - & (Butch) & - & - & - & - & - & birch \\
\hline d & - & ditch & - & - & - & - & (Dutch) & - & - & - & - & - & - & - & - & - \\
\hline g & - & - & - & - & - & - & - & - & - & - & - & - & - & - & - & - \\
\hline p & peach & pitch & - & - & patch & - & - & - & poach & - & pooch & - & - & pouch & - & perch \\
\hline$t$ & teach & - & - & - & - & - & touch & - & - & - & - & - & - & - & - & - \\
\hline k & - & - & - & ketch & catch & - & - & - & coach & - & - & - & - & couch & - & - \\
\hline $\mathrm{m}$ & - & (Mitch) & - & - & match & - & much & - & - & - & mooch & - & - & - & - & - \\
\hline $\mathrm{n}$ & - & niche & - & - & (natch) & notch & - & - & - & - & - & - & - & - & - & - \\
\hline 1 & leech & - & - & letch & latch & - & - & - & loach & - & - & - & - & - & - & lurch \\
\hline I & reach & rich & - & wretch & - & - & - & - & roach & - & - & - & - & - & - & - \\
\hline j & - & - & - & - & - & - & - & - & - & - & - & - & - & - & - & - \\
\hline$z$ & - & - & - & - & - & - & - & - & - & - & - & - & - & - & - & - \\
\hline s & - & - & - & - & - & - & such & - & - & - & - & - & - & - & - & search \\
\hline J & - & - & - & - & - & - & - & - & - & - & - & - & - & - & - & - \\
\hline$v$ & - & - & - & - & - & - & - & - & - & - & - & - & - & vouch & - & - \\
\hline $\mathbf{f}$ & - & fitch & - & fetch & - & - & - & - & - & - & - & - & - & - & - & - \\
\hline $\mathrm{h}$ & - & hitch & - & - & hatch & - & hutch & - & - & - & - & - & - & - & - & - \\
\hline$\delta$ & - & - & - & - & - & - & - & - & - & - & - & - & - & - & - & - \\
\hline$\theta$ & - & - & - & - & thatch & - & - & - & - & - & - & - & - & - & - & - \\
\hline$w$ & - & witch & - & - & - & watch & - & - & - & - & - & - & - & - & - & - \\
\hline dz & - & - & - & - & - & - & - & - & - & - & - & - & - & - & - & - \\
\hline ts & - & - & - & - & - & - & - & - & - & - & - & - & - & - & - & church \\
\hline (ø) & each & jtch & $\mathbf{H}$ & etch & - & - & - & - & - & - & - & - & - & (ouch) & - & - \\
\hline
\end{tabular}




\begin{tabular}{|c|c|c|c|c|c|c|c|c|c|c|c|c|c|c|c|c|}
\hline \multirow{2}{*}{$\begin{array}{c}\begin{array}{c}\text { First } \\
\text { Consonant }\end{array} \\
\end{array}$} & \multicolumn{16}{|c|}{ Vowel } \\
\hline & $\mathrm{i}$ & I & e & $\varepsilon$ & $æ$ & $\mathbf{a}$ & $\Lambda$ & 0 & 0 & $\mathbf{u}$ & $\mathbf{u}$ & aI & II & au & $\mathrm{ju}$ & 3 \\
\hline & \multicolumn{16}{|c|}{ Words Ending With No Final Consonant } \\
\hline b & bee & - & bay & - & - & - & - & - & bow & - & (boo) & buy & boy & bow & - & bur \\
\hline d & D & - & day & - & - & - & - & daw & doe & - & do & die & - & dhow & - & - \\
\hline $\mathrm{g}$ & - & - & gay & - & - & - & - & - & go & - & goo & guy & (goy) & - & - & - \\
\hline $\mathrm{p}$ & pea & - & pay & - & - & (pa) & - & paw & (Poe) & - & (Pooh) & pie & poi & (pow) & pew & purr \\
\hline$t$ & tea & - & - & - & - & - & - & - & toe & - & two & tie & toy & tau & - & - \\
\hline k & key & - & (Kay) & - & - & - & - & caw & - & - & coup & chi & coy & cow & cue & cur \\
\hline $\mathrm{m}$ & $\mathrm{me}$ & - & may & - & - & (ma) & - & - & mow & - & moo & my & - & - & mew & myrrh \\
\hline $\mathrm{n}$ & knee & - & neigh & - & - & - & - & gnaw & no & - & knew & nigh & - & now & - & - \\
\hline 1 & (Lee) & - & lay & - & - & (la) & - & law & low & - & lieu & lie & - & - & - & - \\
\hline$r$ & - & - & ray & - & - & (rah) & - & raw & row & - & rue & rye & (Roy) & - & - & - \\
\hline $\mathrm{j}$ & (ye) & - & (yay) & - & - & - & - & - & (yo) & - & you & - & - & - & - & - \\
\hline$z$ & $\mathrm{Z}$ & - & - & - & - & - & - & - & - & - & 200 & $\mathrm{xi}$ & - & - & - & - \\
\hline$s$ & see & - & say & - & - & - & - & saw & sew & - & sue & sigh & soy & sow & - & sir \\
\hline $\int$ & she & - & shay & - & - & shah & - & - & show & - & shoe & shy & - & - & - & - \\
\hline v & V & - & - & - & - & - & - & - & - & - & - & vie & - & vow & view & - \\
\hline f & fee & - & (Fay) & - & - & - & - & (faugh) & foe & - & foo & (fie) & - & - & few & fur \\
\hline h & he & - & hay & - & - & $\ldots$ & - & haw & hoe & - & who & high & - & how & hew & her \\
\hline$\gamma$ & thee & - & they & - & - & - & the & - & though & - & - & thy & - & thou & - & - \\
\hline$\theta$ & - & - & - & - & - & - & - & thaw & - & - & - & thigh & - & - & - & - \\
\hline$w$ & we & - & way & - & - & - & - & - & woe & - & $w \infty O$ & why & - & (wow) & - & were \\
\hline$d z$ & $G$ & - & $\mathrm{J}$ & - & - & - & - & jaw & (Joe) & - & (Jew) & - & joy & - & - & - \\
\hline t5 & - & - & - & - & - & - & - & - & - & - & chew & - & - & chow & - & - \\
\hline (ø) & E & - & A & - & - & (ah) & $a$ & (aw) & owe & - & - & 1 & (oy) & (ow) & you & (er) \\
\hline
\end{tabular}

Note: Letters of the alphabet (e.g., Z, E above) represent the spoken forms of the letters ([zi], [i]). Proper nouns, compound words, and slang words appear in parentheses.

(Manuscript received July 17, 1986

revision accepted for publication September 18, 1986.) 